\title{
Presentación del número
}

\section{Presentación del número 2 volumen 6 de RIECS}

\author{
Manuel Rodríguez Zapata \\ Profesor Emérito del Departamento de Medicina y Especialidades Médicas, Universidad de Alcalá; \\ manuel.rodriguezz@uah.es; https://orcid.org/0000-0003-3673-4025
}

DOI: https://doi.org/10.37536/RIECS.2021.6.2.296

El número actual de RIECS culmina sus 6 primeros años de existencia y, a pesar de las vicisitudes que se han producido en estos tiempos, reafirma los objetivos fundacionales de crear un órgano de expresión y comunicación multidisciplinar en los ámbitos de la educación y la comunicación abierto a todos los miembros y a los colectivos que forman, no solo la Facultad de Medicina y Ciencias de la Salud de la Universidad de Alcalá, sino a todos los miembros de la propia Universidad de Alcalá y de otras universidades de ámbito nacional o internacional.

En esta ocasión contamos con la colaboración de distintos autores procedentes de otras universidades nacionales, como la Universidad de Valencia o internacionales, como la Fundación Universitaria de San Gil, Santander Colombia.

En el espacio reservado a los trabajos originales hemos seleccionado el trabajo presentado por Laura Jiménez Reyes et al, del Servicio de Medicina Interna del Hospital Central de la Defensa Gómez Ulla, titulado Antibioterapia inhalada en paciente con bronquiectasias pulmonares e infección bronquial. En el que se apuesta por este tratamiento y se observa una mejora de la calidad de vida y una reducción del número de exacerbaciones y la mortalidad.

María-Alejandra Ortega-Barco, Magister en Enfermería Docente del Programa de Enfermería de la Fundación Universitaria de San Gil, Santander, Colombia. En colaboración con Daniela MarínRodríguez, Secretaría de Salud del Departamento de Santander, Colombia. Nos presentan su trabajo sobre un tema tan de actualidad como Prácticas seguras en administración de medicamentos en un hospital de segundo nivel de atención, concluyendo que el personal de enfermería mostró un cumplimiento de prácticas seguras en la administración de medicamentos.

Uno de los problemas con los que nos encontramos en estos momentos, es la definición de determinados productos (alimento, medicamento, producto sanitario, cosmético y biocida), denominados productos frontera en alguna de las categorías administrativas. En su artículo original, Bernardo Prieto Muñoz y María del Carmen Vidal Miñana, pertenecientes al Departamento de Farmacia y Tecnología Farmacéutica y Parasitología y al Departamento de Medicina Preventiva, Salud Pública, Bromatología, Toxicología y Medicina Legal de la Universidad de Valencia, respectivamente, y titulado Productos frontera. Metodología de clasificación en inspección farmacéutica como garantía de salud pública y buen gobierno, nos informan de los procedimientos para detectar errores de clasificación y fraudes en la comercialización, profundizando en la seguridad de la utilización de estos productos.

Se completa el apartado dedicado a trabajos originales con una aportación sobre educación física y deporte. Sandra José Paticene et al, pertenecientes a la Universidad de Alcalá, en su artículo Estereotipos de género en educación física y deporte en Mozambique, plantean la construcción y la perpetuación del estereotipo sexual de género en la educación física y el deporte en Mozambique. Los resultados indican de la perpetuación de estereotipos patriarcales en este campo y opinan que se hace obligado la implantación de cambios en las estructuras sociales que influyan en la ideología preconcebida de la mujer.

El último de los artículos originales, lo presentan Andrea Revuelta y Juan Carlos Luis Pascual, enfermera del Hospital Universitario de Guadalajara y egresada del Máster Universitario en Acción 
Humanitaria Sanitaria de la UAH y del Director del Departamento de Educación de la Universidad de Alcalá respectivamente, titulado Impacto de un Programa online de Intervención en Mindfulness y Compasión en Profesionales Sanitarios durante la pandemia de la covid-19. Estudio piloto. En el trabajo valoran el impacto de un programa online de intervención de Mindfulness y Compasión en Profesionales Sanitarios en relación con el bienestar y el cansancio emocional realizado mediante un ensayo clínico aleatorizado a través de un reclutamiento electivo.

Incorporamos en este número en la sección "Artículo Especial" que trata sobre un aspecto central de la formación y la educación de nuestros estudiantes que desarrollan su actividad en diferentes ámbitos docentes, como es la "Simulación", a través de la Experiencia del Centro de Simulación de la Facultad de Medicina de la Universidad de Navarra, presentado por Javier Pueyo et al, en el que nos describen los aspectos fundamentales en la concepción, el funcionamiento y la utilidad de un laboratorio de simulación, como en de la Universidad de Navarra, que constituye un ejemplo a seguir en nuestro país.

Publicamos un caso clínico presentado por Pablo Vicente Muñoz-Ramon et al, sobre Imagen multimodal en Epiteliopatía Pigmentosa Placoide Multifocal Posterior Aguda (APMPPE), que ilustra el proceso diagnóstico de esta entidad nosológica y la posible asociación con diferentes enfermedades a nivel sistémico, así como una extensa revisión bibliográfica.

En este número, hemos planteado dos artículos de opinión sobre dos aspectos de especial importancia y actualidad. La formación clínica en el seno de la pandemia por COVID-19 y el debate sobre los cuidados al final de la vida.

Juan Ángel Muñoz López, profesor del Departamento de Enfermería y Fisioterapia de la Universidad de Alcalá y Vicedecano Adjunto de Enfermería del Hospital Príncipe de Asturias de Alcalá de Henares, profundiza en su artículo de opinión, Prácticas clínicas en época de pandemia, en las estrategias utilizadas durante la pandemia para mantener las prácticas clínicas de los estudiantes de Enfermería, nuestro autentico futuro.

Los profesores asociados en Ciencias de la Salud del Departamento de Medicina y Especialidades Médicas de la Universidad de Alcalá, Antonio Sacristán Rodea y Miguel Ferrari Sanjuan, en su artículo de opinión Tratamientos al final de la vida: cuidados paliativos, sedación terminal, eutanasia y suicidio medicamente asistido (SMA), plantean un tema tan crucial como es el manejo del paciente al final de la vida y nos ilustran sobre los fenómenos sociosanitarios nuevos como son cuidados paliativos, la sedación terminal, la eutanasia y el SMA.

Hemos reservado este espacio, especial por su trascendencia académica y por su interés, denominado Aula Magna, a una excelente colaboración de la Profesora Titular de Lengua Española y actual Decana de la facultad de Filosofía y Letras de la Universidad de Alcalá, Silvia Gumiel Molina, titulado Ni tan de ciencias ni tan de letras. La necesidad de la interdisciplinariedad. Un ejemplo tomando en consideración la medicina y la lingüística, en el que reflexiona brillantemente sobre, como la formación lingüística puede contribuir al progreso en la formación de estudiante de medicina, y señala los aspectos, como la adquisición de la lengua materna o la relación entre lenguaje y pensamiento, que deben ser de obligado conocimiento para el médico futuro.

En el espacio Cartas al Editor, Laura Mola Reyes et al, miembros de los Servicios de Endocrinología y Nutrición y de Medicina Nuclear del Hospital Central de la Defensa Gómez Ulla y del Instituto de Investigación I+12-Hospital Universitario 12 de Octubre, nos presentan los principales hallazgos de su estudio Utilidad de la gammagrafía de captación tiroidea en enfermedad tiroidea no maligna

Completamos el presente número con un Revisión Histórica, en la que José Antonio Martínez Pérez, en su artículo El divino sordo, nos ilustra sobre la biografía, la trascendencia y la enfermedad que padeció uno de los más grandes maestros del clasicismo musical y una figura relevante y singularmente influyente en el mundo cultural de su tiempo, cuando apenas se han cumplido los doscientos años de su nacimiento. 
Finalmente llegamos a nuestro espacio preferido, Ágora, que en esta ocasión presenta un relato corto de una colaboradora habitual de la revista. La profesora Consuelo Giménez Pardo, titulado "Por amabilidad".

Como siempre, espero que les guste.

(C) 2021 por los autores; Esta obra está sujeta a la licencia de Reconocimiento 4.0 Internacional de Creative Commons. Para ver una copia de esta licencia, visite http://creativecommons.org/licenses/by-nc-nd/4.0/. 